\title{
Energy funneling in a bent chain of Morse oscillators with long-range coupling
}

\author{
Larsen, Peter Ulrik Vingaard; Christiansen, Peter Leth; Bang, Ole; Archilla, J.F. R.; Gaididei, Yuri B.
}

Published in:

Physical Review E. Statistical, Nonlinear, and Soft Matter Physics

Link to article, DOI:

10.1103/PhysRevE.69.026603

Publication date:

2004

Document Version

Publisher's PDF, also known as Version of record

Link back to DTU Orbit

Citation (APA):

Larsen, P. U. V., Christiansen, P. L., Bang, O., Archilla, J. F. R., \& Gaididei, Y. B. (2004). Energy funneling in a bent chain of Morse oscillators with long-range coupling. Physical Review E. Statistical, Nonlinear, and Soft Matter Physics, 69(2), 026603. https://doi.org/10.1103/PhysRevE.69.026603

\section{General rights}

Copyright and moral rights for the publications made accessible in the public portal are retained by the authors and/or other copyright owners and it is a condition of accessing publications that users recognise and abide by the legal requirements associated with these rights.

- Users may download and print one copy of any publication from the public portal for the purpose of private study or research.

- You may not further distribute the material or use it for any profit-making activity or commercial gain

- You may freely distribute the URL identifying the publication in the public portal 


\title{
Energy funneling in a bent chain of Morse oscillators with long-range coupling
}

\author{
P. V. Larsen* and P. L. Christiansen \\ Informatics and Mathematical Modelling, Technical University of Denmark, DK-2800 Kgs. Lyngby, Denmark \\ O. Bang \\ Informatics and Mathematical Modelling, Technical University of Denmark, DK-2800 Kgs. Lyngby, Denmark \\ and Research Center COM, Technical University of Denmark, DK-2800 Kgs. Lyngby, Denmark \\ J. F. R. Archilla \\ Departamento de Fisica Aplicada I, Universidad de Sevilla, Avda. Reina Mercedes s/n, 41012 Sevilla, Spain \\ Yu. B. Gaididei \\ Bogolyubov Institute for Theoretical Physics, 03143 Kiev, Ukraine
}

(Received 13 October 2003; published 17 February 2004)

\begin{abstract}
A bent chain of coupled Morse oscillators with long-range dispersive interaction is considered. Moving localized excitations may be trapped in the bending region. Thus chain geometry acts like an impurity. An energy funneling effect is observed in the case of random initial conditions.
\end{abstract}

DOI: 10.1103/PhysRevE.69.026603

PACS number(s): 05.45.Yv, 63.20.Ry, 63.20.Pw, 87.15.Aa

\section{INTRODUCTION}

Nonlinear excitations (solitons, discrete breathers, intrinsic localized modes, etc.) have been drawing increasing attention over recent years and are widely believed to be responsible for several effects in molecular chains, such as charge and thermal conductivity, energy transfer and localization, etc. (see the reviews in Refs. [1-6] e.g.).

Initially, the geometrical features of the polymers and biopolymers were essentially neglected and energy transfer and localization was mostly attributed to inhomogeneities and impurities [7-12] or nonlinear excitations [8,13-17]. Also, discreteness plays an important role in the localization of these excitations. The inhomogeneities have been modeled by different masses at various chain sites $[7,10,12]$, by changes in the coupling between molecular sites $[7,8]$, or by different on-site potentials [11] as well as conformational defects [17]. In general, impurities have been shown to act as filters governing the progression of incoming excitations. Thus both reflection, trapping and transmission of incident moving discrete breathers through the impurity region can occur $[7,8,10-12,17]$. Similar effects have been observed through collisions between moving discrete breathers; thus Refs. $[8,14,16]$ showed how stationary large amplitude discrete breathers, on the average, absorb energy from colliding breathers of smaller amplitude. Thus the large amplitude breather may play a role similar to that of an impurity [12].

Recently, both long-range dipole-dipole interaction $[18,19]$, helicity $[20,21]$, and curvature [22-24] have been included in the nonlinear transport theory, as well as combinations of these effects [25-29]. It has been shown that chain geometry induces effects similar to those of impurities [2225].

Special attention has been paid to models of biological

*Electronic address: pvl@imm.dtu.dk macromolecules, such as proteins $[1,3,13]$ and DNA [1,9$11,13,15,30]$. These are obvious choices for more complex geometric models, as their structure is crucial for their functionality [31,32]. A widely used model was presented by Peyrard and Bishop [33] in the context of statistical mechanics, where the solutions are shown to possess a self-focusing effect, which is viewed as a precursor for thermal denaturation. In biological environments, thermal fluctuations are always present and have been considered in Refs. [9,30,3436], for example. In these references, it was shown that solitons or discrete breathers can be generated from initial random thermal fluctuations.

The aim of the present work is to study the interplay between chain geometry and long-range interaction in an augmented Peyrard-Bishop model. We show how a new mechanism for energy accumulation in the systemfunneling - may be provided by the geometry of the chain. We consider a simple approximative description of the longrange intersite coupling by modeling it as a dipole-dipolelike interaction. Using an attractive long-range interaction, we study the effect of the geometry of the bent chain on the dynamics. The particular shape of the bend turns out to make no qualitative difference in terms of trapping and funneling. We therefore choose a simple wedge-shaped geometry. As initial conditions we use discrete breathers as well as randomly distributed fluctuations $[9,14]$.

In Sec. II we introduce the model, which includes the long-range interactions and the chain geometry. In Sec. III, we investigate breather dynamics in the system and in Sec. IV random initial conditions are considered. Section V summarizes our results and contains a discussion.

\section{MODEL}

We consider a one-dimensional lattice of Morse oscillators with the Hamiltonian density 


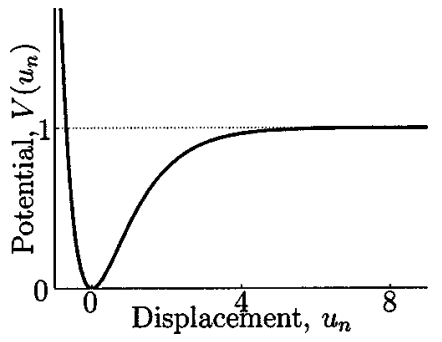

FIG. 1. The Morse potential, $V\left(u_{n}\right)=\left[\exp \left(-u_{n}\right)-1\right]^{2}$.

$$
\begin{aligned}
\mathcal{H}_{n}= & \frac{1}{2} \dot{u}_{n}^{2}+\frac{C}{2}\left(u_{n}-u_{n-1}\right)^{2}+\left[\exp \left(-u_{n}\right)-1\right]^{2} \\
& -\frac{1}{2} \sum_{m}{ }^{\prime} J_{n m} u_{n} u_{m},
\end{aligned}
$$

where the prime indicates $m \neq n$ in the summation. The Hamiltonian for the system becomes $H=\sum_{n=-N}^{n=N} \mathcal{H}_{n}$, where the total number of sites is $N_{T}=2 N+1$. In Eq. (1) the first term is the kinetic energy at the $n$th site. Then follows a harmonic potential interaction between neighboring sites, $C$ being the dispersion parameter. An on-site Morse potential (shown in Fig. 1) describes the atomic interaction. Finally, there is a summation of long-range dipole-dipole-like interactions in which the coefficients are given by

$$
J_{n m}=\frac{J_{0}}{\left|\boldsymbol{r}_{n}-\boldsymbol{r}_{m}\right|^{3}}
$$

where $J_{0}$ is a strength parameter, and $\mathbf{r}_{n}$ denotes the position of the $n$th site. In our model the distance between neighboring sites, $\left|\boldsymbol{r}_{n+1}-\boldsymbol{r}_{n}\right|$, is constant and normalized to unity.

From the Hamiltonian we get the equations of motion

$$
\begin{aligned}
\ddot{u}_{n}+ & C\left(2 u_{n}-u_{n-1}-u_{n+1}\right)-2 e^{-u_{n}\left[e^{-u_{n}}-1\right]} \\
& -\sum_{m}{ }^{\prime} J_{n m} u_{m}=0 .
\end{aligned}
$$

At the ends of the molecule we use the free boundary conditions

$$
\begin{gathered}
u_{-N-1}=u_{-N}, \\
u_{N+1}=u_{N} .
\end{gathered}
$$

The wedge-shaped chain is given by

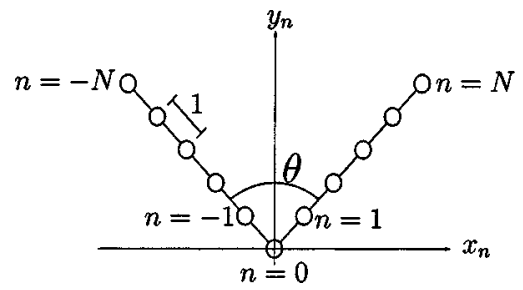

FIG. 2. Wedge chain with opening angle, $\theta$.
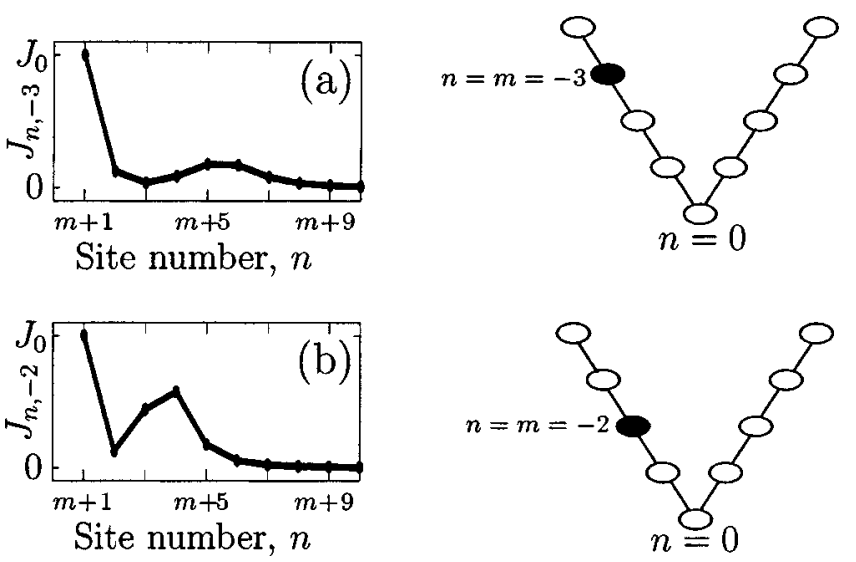

FIG. 3. Long-range interaction coefficients $J_{n m}$ for fixed (a) $m$ $=-3$ and (b) $m=-2 . \theta=35^{\circ}$.

$$
\boldsymbol{r}_{n}=\left(x_{n}, y_{n}\right)=\left(n \sin \frac{\theta}{2},|n| \cos \frac{\theta}{2}\right),
$$

where $\theta$ denotes the fixed wedge angle (see Fig. 2). We note that the geometry of the chain only comes into play through the long-range interactions. In fact, earlier studies of the long-range effect in curved molecular chains show that the exact form of the additional dispersion is not crucial as long as it decreases rapidly with distance $[15,28]$. Throughout the paper we use $C=0.075$ (a typical value for DNA $[19,29,34]$ ) and $J_{0}=0.5$ (based on estimates of the dipole moment [19]).

Figure 3 gives a detailed picture of the long-range interaction by plotting the interaction coefficient $J_{n m}$ versus $n$, for fixed (a) $m=-3$ and (b) $m=-2$. We see that the closer $m$ gets to the bend at $n=0$, the higher the shoulder in the $J_{n m}$-profile becomes. This feature suggests an analogy in which the bend acts as an impurity. Away from the bend this effect rapidly drops off.

The long-range interaction in curved chains may be represented as follows:

$-\sum_{n} \sum_{m}{ }^{\prime} J_{n m} u_{n} u_{m}=\frac{1}{2} \sum_{n} \sum_{m}{ }^{\prime} J_{n m}\left(u_{n}-u_{m}\right)^{2}+\sum_{n} V_{n}^{\mathrm{Eff}} u_{n}^{2}$,

where the first summations on the right hand side correspond to the inhomogeneous dispersion (seen in Fig. 3), while the second summation, in which $V_{n}^{\mathrm{Eff}} \equiv-\Sigma_{m}^{\prime} J_{n m}$ is introduced, corresponds to an effective on-site potential [28]. The potential $V_{n}^{\text {Eff }}$ has the double-well profile, shown in Fig. 4. In the

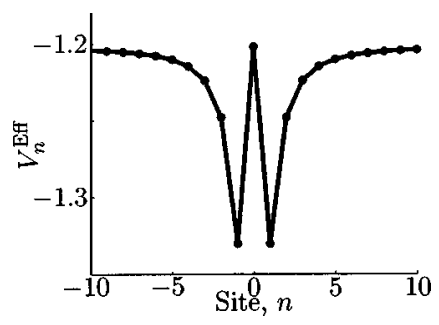

FIG. 4. Effective potential, $V_{n}^{\mathrm{Eff}} \equiv-\sum_{m}^{\prime} J_{n m}$, versus site number, $n$. 

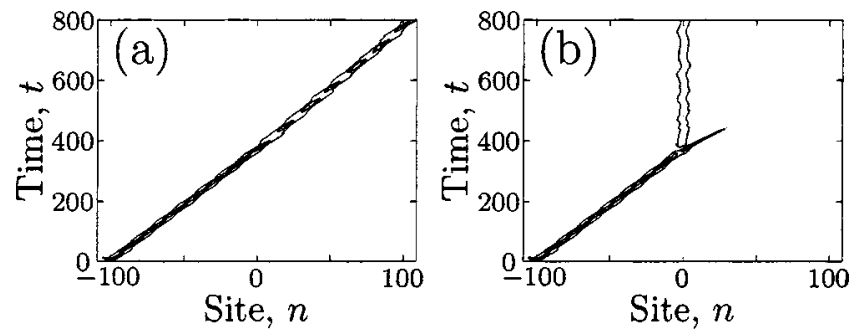

FIG. 5. Contour plots for the evolution of the Hamiltonian density, $\mathcal{H}_{n}$, for $N_{T}=301, J_{0}=0.5, v=0.2, k=0.2, A=0.5$, and $\nu=-100$, yielding $H=0.13$. Five equidistant contours $H$ $=0.005 \ldots 0.05$. (a) $\theta=140^{\circ}$ (transmission). (b) $\theta=95^{\circ}$ (trapping).

following we shall see how the impurity, or effective on-site potential, introduced by the bend can reflect, trap or transmit incoming excitations.

\section{ENERGY TRAPPING}

In this Section we consider the interaction between the bend and the incoming localized excitations. A fourth order Runge-Kutta solver is used to simulate Eq. (1) on a chain with $N_{T}=301$ sites. A stepsize in time of 0.005 ensures conservation of the Hamiltonian to a relative accuracy of $10^{-10}$ throughout. We use a Gaussian initial condition

$$
u_{n}(t)=A \exp \left[-k((n-\nu)-v t)^{2}\right]
$$

where site $\nu$ denotes the initial position of the center of mass.

In the following we use the velocity $v=0.2$, the width $k$ $=0.2$, and the amplitude $A=0.5$, because they turn out to provide the right balance between nonlinearity and dispersion to allow the initial condition to evolve rapidly into a discrete moving breather. Insertion of Eq. (6) with these parameter values into the total Hamiltonian gives $H=0.13$. After some initial radiation, the moving breather turns out to possess the energy, $H_{s} \approx 0.08$.

In the following sections we present the numerical simulations of the chain dynamics.

\section{A. Breather dynamics}

In Fig. 5 we show contour plots for the evolution of the Hamiltonian density. A weak bend with $\theta=140^{\circ}$ has no noticeable effect on the breather [Fig. 5(a)] and only a slight decrease of the velocity after passage of the center region is
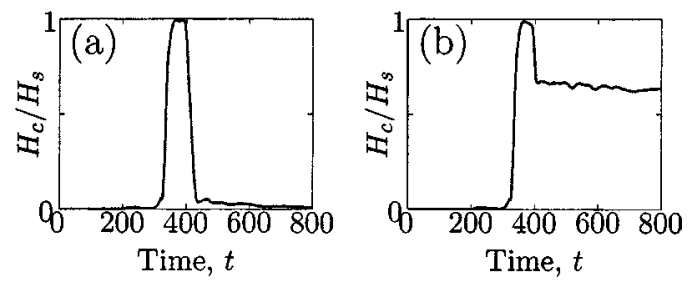

FIG. 6. Relative central energy $H_{c} / H_{s}$ vs time for the simulations in Fig. 5. (a) $\theta=140^{\circ}$. (b) $\theta=95^{\circ}$.

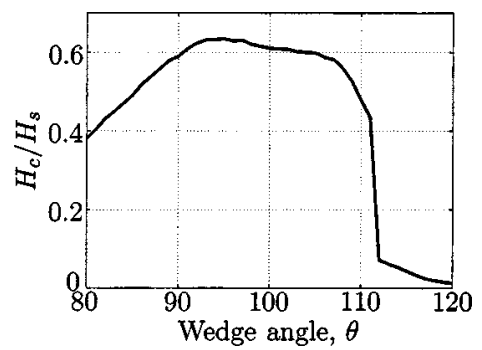

FIG. 7. Relative central energy $H_{c} / H_{s}$ at $t=800$ vs wedge angle $\theta$. System parameters as in Fig. 5.

observed. In contrast for a stronger bend, $\theta=95^{\circ}$, a considerable part of the excitation is trapped at the tip of the wedge $n=0$.

Very strong bends (smaller wedge angles) turn out to result in reflection of the incident breathers. Such scattering properties and their dependence on the strength of the bendinduced impurity, are similar to those of a linear impurity [37] and those of large amplitude breathers acting as an effective impurity [14]. We stress that the specific shape of the bend does not affect the scattering properties of the bent chain. Thus similar properties were observed in a parabolic chain [38].

To analyze the processes in detail, we calculate the central energy, $H_{c}$, in 21 sites around $n=0$ (21 being a typical span of the denaturation bubble of the DNA molecule $[21,34]$ )

$$
H_{c}=\sum_{n=-10}^{10} \mathcal{H}_{n},
$$

where $\mathcal{H}_{n}$ is given by Eq. (1). The results are shown in Fig. 6. In the transmission case with a small bend [Fig. 6(a)], nearly all the energy leaves the region. In the trapping case with a stronger bend [Fig. 6(b)], the trapped energy is stabilized at about $H_{c} / H_{s} \approx 64 \%$. Thus only part of the energy is trapped.

The trapped energy portion, $H_{c} / H_{s}$, calculated at time $=800$ is shown in Fig. 7 as a function of the wedge angle, $\theta$. For smaller and larger $\theta$-values, the energy is lost through reflection and transmission, respectively. Efficient trapping is found for intermediate wedge angles, $90^{\circ}<\theta<107^{\circ}$. The optimal wedge angle for trapping is seen to be around $\theta=95^{\circ}$.

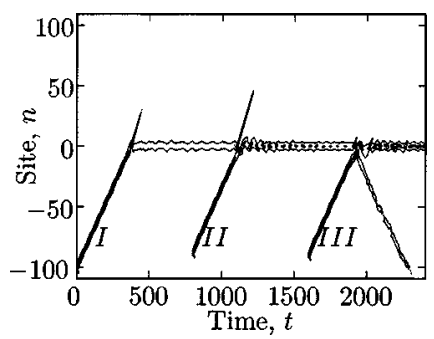

FIG. 8. Trapping of breathers $I, I I$, and $I I I$ at $n=0$. Contour plot for the evolution of the Hamiltonian density, $\mathcal{H}_{n}$, with 5 equidistant lines $H=0.005 \ldots 0.05 . \quad N_{T}=301, J_{0}=0.5, v=0.2, k$ $=0.2, A=0.5$. I: $\nu=-100$ at $t=0 ; I I: \nu=-87$ at $t=800 ; I I I: \nu$ $=-89 t=1600$. 


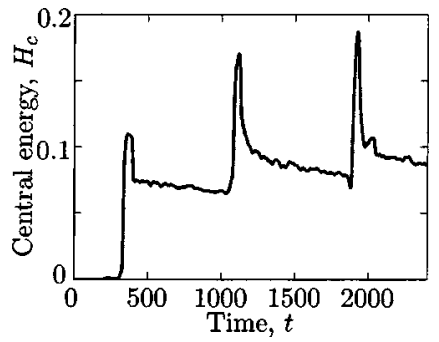

FIG. 9. Central energy $H_{c}$ vs time corresponding to Fig. 8.

\section{B. Multiple breather dynamics}

In Fig. 8 we show the trapping of multiple breathers. A first Gaussian pulse $(I)$ is launched at site $\nu=-100$ at $t$ $=0$. At $t=800$ both the displacements and the velocities are set to zero, $u_{n}(800)=0$ and $\dot{u}_{n}(800)=0$, outside the bent region, $|n|>15$, to remove radiation. This "cleaned" chain is now used as an initial condition for a new simulation, in which we add a second identical Gaussian pulse (II) launched at site $\nu=-87$. Like in other systems the interaction between two breathers, or a breather and an impurity, depends strongly on the relative phase. We choose $\nu=-87$ for the launching of this second pulse to obtain maximal trapping. Using the same procedure, a third identical Gaussian pulse $(I I I)$ is launched at $t=1600$, now at $\nu=-89$. As seen in Fig. 8 we essentially succeed in trapping three breathers at the tip of the wedge chain. Some energy transmission is observed when breathers $I$ and $I I$ are trapped, while reflection occurs at the trapping of breather III. By successively handling the initial conditions as described above, we avoid radiation which, when reflected at the boundaries, distorts the numerical simulations.

The corresponding energy evolution for the central sites is shown in Fig. 9. The ability of the system to trap energy at the bending region is evident, even though more radiation is observed as the number of trapped breathers increases. As noted also in connection with Fig. 6(b), the first incident breather, $I$, loses about $36 \%$ of the total energy before trapping. For the following breathers, $I I$ and $I I I$, both of the corresponding losses amount to $50 \%$. Thus the possibility for trapping more energy at the chain bend by additional incoming breathers may seem exhausted due to an effective saturation.

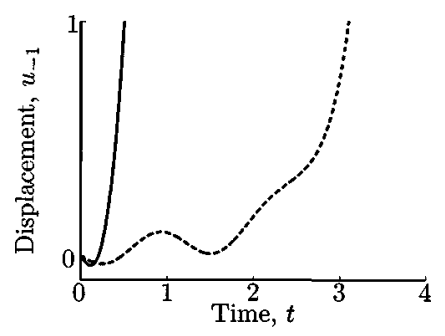

FIG. 10. Displacement at site $n=-1, u_{-1}$, vs time on a bent chain with $N_{T}=99$ and $\theta=45^{\circ}$ for random initial velocities generated with normal distribution: $\left\langle\dot{u}_{n}\right\rangle=0$ and $\sigma_{u_{n}}=1.156$, or $T$ $\approx 310 \mathrm{~K}$ (solid) and $\sigma_{u_{n}}=0.17$, or $T \approx 7 \mathrm{~K}$ (dashed). Initial displacements: $u_{n}=0$ for all $n$.

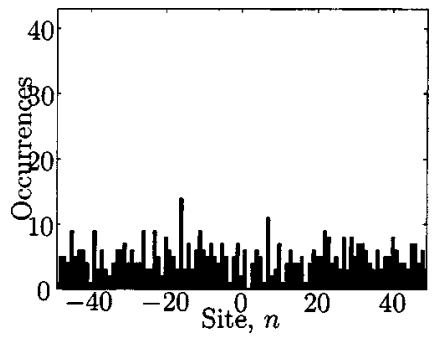

FIG. 11. Straight chain, $\theta=180^{\circ}, N_{T}=99$. Occurrence of amplitudes above threshold, $u_{n}>10$, vs site, $n$, up to a maximum $t$ $=10000$. Initial displacements: $u_{n}=0$ for all $n .500$ random initial velocities with normal distribution: $\left\langle\dot{u}_{n}\right\rangle=0$ and $\sigma_{\dot{u}_{n}}=1.156, T$ $\approx 310 \mathrm{~K}$.

\section{FUNNELING}

The trapping of breathers observed in Sec. III suggests that the bend may funnel energy from the surrounding region. In order to study this in detail, we now explore the dynamics of the chain in the case of random initial conditions. 500 different realizations with zero displacement, $u_{n}$ $=0$, and velocities normally distributed with zero mean, $\left\langle\dot{u}_{n}\right\rangle=0$ and standard deviation $\sigma_{\dot{u}_{n}}=1.156$ are used, corresponding to a temperature of about $37^{\circ} \mathrm{C}$.

Random initial disturbances may create nonlinear localized excitations, interacting with each other and with the effective inhomogeneity caused by the bend $[9,30,34-36]$. In Ref. [9], the number of generated solitons were found to depend on the temperature of the system, $T$, by the power $1 / 3$. Here we find that collision of the nonlinear excitations may result in exponential growth of the oscillation amplitude at the collision site. We observe this phenomenon in Fig. 10 in a bent chain. Here, a sudden increase of the amplitude of the left center site, $u_{-1}$, is observed for two different temperatures. For very small temperature ( $T \approx 7 \mathrm{~K}$, dashed line), the system exhibits longer life time before the oscillation increases exponentially compared to a physiological temperature ( $T \approx 310 \mathrm{~K}$, solid line). This unbounded growth of amplitude implies energy localization at the site.

Our results for a straight chain are depicted in Fig. 11 showing a histogram of the occurrences of sites with a displacement above the threshold value $u_{n}=10$, corresponding to the value for DNA opening used in Ref. [39]. The simu-

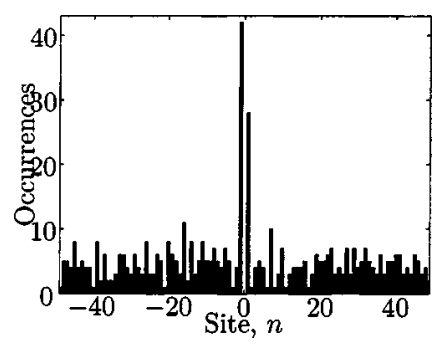

FIG. 12. Wedge chain, $\theta=45^{\circ}, N_{T}=99$. Occurrence of amplitudes above threshold, $u_{n}>10$, vs site, $n$, up to a maximum $t$ $=10000$. Initial displacements: $u_{n}=0$ for all $n$. 500 random initial velocities with normal distribution: $\left\langle\dot{u}_{n}\right\rangle=0$ and $\sigma_{\dot{u}_{n}}=1.156 ; T$ $\approx 310 \mathrm{~K}$. 
lations were discontinued when this threshold value was exceeded. These threshold transgressions, which in most cases occur after a few time units, are seen to be uniformly distributed along the chain.

On a wedge chain with bending angle $\theta=45^{\circ}$, identical initial conditions give the remarkably different result shown in Fig. 12. We see that 70 (out of 500) threshold transgressions occur at the sites $n=-1$ and 1 , between which the long-range interaction is most strongly increased due to the bending. Thus energy localization, implied by unbounded growth of amplitude, is observed in the vicinity of the tip of the wedge which therefore acts as an energy funnel.

\section{CONCLUSION}

On a bent chain of Morse oscillators we find that moving discrete breathers may be trapped at a bending point in the presence of dipole-dipole-like long-range interaction. Thus the role of the geometry for the dynamics is analogous to that of an inhomogeneity. At the bending point, several incident discrete breathers may be trapped. However, energy is lost to radiation and a saturation effect seems to limit the total trapped energy in the vicinity of a given bending point.

For random initial conditions modeling thermal fluctuations, the tendency to unbounded amplitude growth in the vicinity of the bending point is substantially amplified. Thus energy localization is implied in this region which therefore acts as an energy funnel.

The use of a nonlinear potential is crucial for obtaining the energy funneling effect in our model, since no amplitude growth is observed in a linear approximation. The plateau of the characteristic Morse potential allows for the breaking of the hydrogen bonds, e.g., in the molecule to be modeled. In contrast, a linear approximation with a parabolic potential produces too powerful an attraction for this effect to take place and would therefore not be an adequate description of chemical bonds.

We also note that the attracting nature of the long-range interaction is crucial for the occurrence of amplitude growth. Ongoing work on this effect uses a more accurate dipoledipole interaction term and includes the effect of the twisting of the dipoles occurring along the strands of DNA molecules, for example.

\section{ACKNOWLEDGMENTS}

The authors wish to thank S.F. Mingaleev for helpful and inspiring contributions. Yu.B.G. thanks Informatics and Mathematical Modelling, Technical University of Denmark for hospitality. O.B. acknowledges support from the Danish Technical Research Council (Grant No. 26-00-0355). P.V.L. thanks the Oticon Fondation for a study grant. The work was supported by LOCNET Project No. HPRN-CT-1999-00163.
[1] A.S. Davydov, Solitons in Molecular Systems (Reidel, Dordrecht, 1985).

[2] A.J. Heeger, S. Kivelson, J.R. Schrieffer, and W.P. Su, Rev. Mod. Phys. 60, 781 (1988).

[3] A.C. Scott, Phys. Rep. 217, 1 (1992).

[4] Nonlinear Excitations in Biomolecules, edited by M. Peyrard (Springer, Les Ulis, 1995).

[5] S. Flach and C.R. Willis, Phys. Rep. 295, 181 (1998).

[6] L.V. Yakushevich, Nonlinear Physics of DNA (Wiley, New York, 1998).

[7] I. Bena, A. Saxena, G.P. Tsironis, M. Ibañes, and J.M. Sancho, Phys. Rev. E 67, 037601 (2003).

[8] K. Forinash, T. Cretegny, and M. Peyrard, Phys. Rev. E 55, 4740 (1997).

[9] V. Muto, A.C. Scott, and P.L. Christiansen, Phys. Lett. A 136, 33 (1989); Physica D 44, 75 (1990); V. Muto, Nanobiology 1, 325 (1992).

[10] J.J.-L. Ting and M. Peyrard, Phys. Rev. E 53, 1011 (1996).

[11] J. Cuevas, F. Palmero, J.F.R. Archilla, and F.R. Romero, J. Phys. A 35, 10519 (2002).

[12] K. Forinash, M. Peyrard, and B. Malomed, Phys. Rev. E 49, 3400 (1994).

[13] H. Feddersen, Phys. Lett. A 154, 391 (1991).

[14] O. Bang and M. Peyrard, Physica D 81, 9 (1995); Phys. Rev. E 53, 4143 (1996).

[15] S.F. Mingaleev, P.L. Christiansen, Yu.B. Gaididei, M. Johansson, and K.Ф. Rasmussen, J. Biol. Phys. 25, 41 (1999).

[16] T. Dauxois and M. Peyrard, Phys. Rev. Lett. 70, 3935 (1993).

[17] F. Zhang, M.A. Collins, and Yu.S. Kivshar, Phys. Rev. E 51, 3774 (1995).
[18] L. Cruzeiro-Hansson, Phys. Lett. A 249, 465 (1998).

[19] J. Cuevas, J.F.R. Archilla, Yu.B. Gaididei, and F.R. Romero, Physica D 163, 106 (2002).

[20] M. Barbi, S. Cocco, and M. Peyrard, Phys. Lett. A 253, 358 (1999).

[21] M. Barbi, S. Cocco, M. Peyrard, and S. Ruffo, J. Biol. Phys. 24, 97 (1999).

[22] S.F.M. Mingaleev, Yu.B. Gaididei, P.L. Christiansen, and Yu.S. Kivshar, Europhys. Lett. 59, 403 (2002).

[23] R. Reigada, J.M. Sancho, M.I. Ibañes, and G.P. Tsironis, J. Phys. A 34, 8465 (2001).

[24] P.L. Christiansen, Yu.B. Gaididei, and S.F. Mingaleev, J. Phys.: Condens. Matter 13, 1181 (2001).

[25] J.F.R. Archilla, Yu.B. Gaididei, P.L. Christiansen, and J. Cuevas, J. Phys. A 35, 8885 (2002).

[26] B. Sánchez-Rey, J.F.R. Archilla, F. Palmero, and F.R. Romero, Phys. Rev. E 66, 017601 (2002).

[27] J.F.R. Archilla, P.L. Christiansen, S.F. Mingaleev, and Yu.B. Gaididei, J. Phys. A 34, 6363 (2001).

[28] Yu.B. Gaididei, S.F. Mingaleev, and P.L. Christiansen, Phys. Rev. E 62, R53 (2001).

[29] J. Cuevas, F. Palmero, J.F.R. Archilla, and F.R. Romero, Phys. Lett. A 299, 221 (2002).

[30] T. Dauxois, M. Peyrard, and C.R. Willis, Physica D 57, 267 (1992).

[31] W. Saenger, Principles of Nucleic Acid Structure (SpringerVerlag, New York, 1984).

[32] C.R. Calladine and H.R. Drew, Understanding DNA (Academic Press, London, 2002). 
[33] M. Peyrard and A.R. Bishop, Phys. Rev. Lett. 62, 2755 (1989).

[34] T. Dauxois, M. Peyrard, and A.R. Bishop, Phys. Rev. E 47, 684 (1993).

[35] G.P. Tsironis, A.R. Bishop, A.V. Savin, and A.V. Yolotaryuk, Phys. Rev. E 60, 6610 (1999).
[36] M. Peyrard, Physica D 160, 184 (1998).

[37] A.A. Sukhorukov, Yu.S. Kivshar, O. Bang, J.J. Rasmussen, and P.L. Christiansen, Phys. Rev. E 63, 036601 (2001).

[38] P.V. Larsen, IMM-THESIS-2002-32, Technical University of Denmark, Lyngby, 2002.

[39] S. Cocco and R. Monasson, J. Chem. Phys. 122, 10017 (2000). 